\title{
Radioactive Iodine Therapy for Differentiated Thyroid Cancer: Lessons from Confronting Controversial Literature on Risks for Secondary Malignancy
}

\author{
Mark Tulchinsky ${ }^{1}$, Ina Binse ${ }^{2}$, Alfredo Campennì ${ }^{3}$, Sabina Dizdarevic ${ }^{4}$, Luca Giovanella ${ }^{5}$, Ian Jong ${ }^{6}$, Kalevi Kairemo ${ }^{7}$, \\ and Chun K. Kim ${ }^{8}$ \\ ${ }^{1}$ Section of Nuclear Medicine, Department of Radiology, Pennsylvania State University, Hershey, Pennsylvania; ${ }^{2}$ Department of \\ Nuclear Medicine, University Hospital Essen, Essen, Germany; ${ }^{3}$ Nuclear Medicine Unit, Department of Biomedical and Dental \\ Sciences and Morphofunctional Imaging, University of Messina, Messina, Italy; ${ }^{4}$ Imaging and Nuclear Medicine Department, \\ Brighton and Sussex University Hospitals, Brighton and Sussex Medical School, Brighton, United Kingdom; ${ }^{5}$ EOC Thyroid Diagnosis \\ and Therapy Centre, Nuclear Medicine and PET/CT Centre, Oncology Institute of Southern Switzerland, Bellinzona and Lugano, \\ Switzerland; ${ }^{6}$ Nuclear Medicine Department, Monash Health, Melbourne, Victoria, Australia; ${ }^{7}$ Docrates Cancer Center, Molecular \\ Radiotherapy and Nuclear Medicine, Helsinki, Finland; and ${ }^{8}$ Department of Nuclear Medicine, Hanyang University College of \\ Medicine, Seoul, Korea
}

$\mathbf{T}$ here has been increasing focus on the side effects of radioactive iodine treatment (RAIT) given to patients with well-differentiated thyroid cancer (WDTC). The secondary malignancies evoke a particularly high level of concern in patients and health-care professionals because of potential grave consequences. A recent report by Molenaar et al. aimed to clarify the risks of secondary hematologic malignancy (1). The investigators purported to show that RAIT was associated with an increased risk of acute and chronic myeloid leukemia. Furthermore, they made the case for an accelerated clinical course in patients who developed acute myeloid leukemia after RAIT. They concluded with a recommendation that RAIT "should be limited to patients with high-risk disease features, and patients with WDTC treated with adjuvant [radioactive iodine] should be monitored for myeloid malignancies as part of cancer surveillance." This recommendation is counter to the standard of practice and implies an escalation in financial and emotional burdens on these patients. In the following deliberations, we intend to show that the recommendation by Molenaar et al. is unjustified, and we will outline some of the important lessons for the nuclear medicine specialists (NMSs).

The article was published online on December 18, 2017, in the Journal of Clinical Oncology (JCO), which is ranked in the top $1 \%$ of oncology journals. About a week later, the article was read by a NMS who has a particular interest in RAIT. Interestingly, there was no mention of this article in the selected readings or news releases distributed by the relevant societies. An international group of NMSs quickly brought the article to the attention of the leadership at the Society of Nuclear Medicine and Molecular Imaging and the American College of Nuclear Medicine (ACNM). Most journals provide a

Received Mar. 11, 2018; revision accepted Mar. 26, 2018.

For correspondence or reprints contact: Mark Tulchinsky, Pennsylvania State University, Section of Nuclear Medicine, Department of Radiology, 500 University Dr., Hershey, PA 17033.

E-mail: mark.tulchinsky@gmail.com

Published online Apr. 13, 2018.

COPYRIGHT (C) 2018 by the Society of Nuclear Medicine and Molecular Imaging. DOI: 10.2967/jnumed.118.211359 short time limit for accepting letters to the editor about an article; for $J C O$, this limit is $6 \mathrm{wk}$ from the date that the article appears online. This fortuitous early identification of the article allowed the NMS group to submit to the JCO Editor-in-Chief a petition endorsed by 67 RAIT experts from around the globe asking for the article to be retracted. An additional request for the article retraction was submitted by the ACNM. Because of these efforts, a commentary by the Society of Nuclear Medicine and Molecular Imaging and several letters to the editor are expected to appear in the same issue of $J C O$ as the article, as well as possibly in other issues. The first lesson to take from this experience should be that identification of potentially practice-changing articles is too important to be left to chance and should rapidly evolve into a systematic process.

Molenaar et al. built the study cohort (148,215 patients with WDTC) from the Surveillance, Epidemiology, and End Results Program database (1). The authors state that "a large population with relatively homogenous treatment exposure" constituted the main strength of their study. A sophisticated statistical methodology (some of it recently developed by the same group) was applied to investigate the risks of developing individual subtypes within secondary hematologic malignancy. As the discussions among the NMS group progressed, it became clear that, in general, we had inadequate understanding of and experience with large population databases. In fact, none of the recent major publications dealing with analyses of such databases included NMSs as authors. Hence, the second lesson focuses on a substantial knowledge gap that calls on development of educational and research programs, through the Society of Nuclear Medicine and Molecular Imaging and the ACNM, grooming internal experts in the analysis of these databases. Remaining handicapped in this critical field of research, NMSs will end up following practice recommendations derived from these sources by individuals from other specialties who developed such skills.

The comments of NMSs to JCO emphasized that treatment recommendations should be based on careful analysis of the risks and benefits observed in the same large population. The authors of the article in question (1) took a shortcut, concluding — on the basis of a recent guideline by the American Thyroid Association (2) - that RAIT "has shown no or questionable benefit." A better approach 
would have been for the authors to examine the benefits of RAIT in the same "large population." Specifically, instead of calling the benefit "questionable" in intermediate-risk WDTC, the authors should have accepted the onus of resolving that question by testing it in the same "large population." Importantly, they did not mention a study by Ruel et al. (3) showing a survival benefit for RAIT in intermediate-risk WDTC. Strikingly, they either understated or ignored completely the benefits they observed in the RAIT group. For example, their data showed that RAIT had a protective effect on the risk of developing multiple myeloma. This finding appears in one sentence of the Results and one sentence of the Discussion. The authors qualify this finding as "interesting" but offer no explanation for it, writing instead that "the possible mechanism of [the finding] needs further investigation." An unbiased discussion would have included two plausible explanations: the flawed data and the radiation hormesis. The authors also neglected to mention that in other studies of patients with hyperthyroidism and WDTC, RAIT has shown a protective effect on the risks of developing certain malignancies $(4,5)$.

Another fact the authors neglected to mention is that, according to their own data (inconspicuously placed in Table 2 of the Appendix), the RAIT group had a markedly lower number of solid cancers. Solid tumors were found in $6.45 \%(5,100 / 79,033)$ of patients treated with surgery, as opposed to a significantly lower percentage, $5.60 \%(3,827 / 68,374 ; P<0.00001$, using a $z$ score for 2 population proportions), of patients treated with surgery plus RAIT. Extrapolated to 100,000 cases, RAIT would have added 66 excess cases of acute myeloid leukemia plus chronic myeloid leukemia while reducing solid cancers by 856 cases. We would accept a criticism that this calculation lacks multivariate analysis for confounding factors, but advanced age could have been the only known confounder and the patients in the RAIT group were actually younger than those in the surgery group. Seeing no discussion of this striking beneficial observation should lead to the only logical conclusion that the authors had a strong anti-RAIT bias. Hence, their findings and recommendations must be viewed with an equitable measure of skepticism.

Molenaar et al. conjectured that the "results support using the least effective dose to treat patients who have high-risk features to avoid excess bone marrow exposure" (1). This concept may work for chemotherapy but does not currently apply to RAIT. First, RAIT is not administered as a "dose" but instead is given as a prescribed activity after considering a multitude of clinical and imaging factors to arrive at the amount. The decision is usually an empiric one, guided by more than 70 years of published experience. Second, the argument for "the least effective dose" ignores the fact that studies comparing lower to higher prescribed activities usually do not define the goal of RAIT - ablation versus adjuvant therapy versus therapy of metastatic disease (2). This trinary categorization of therapy is typically not clearly stated either to the patients or in contemporary practice. The authors lump the 3 categories of RAIT into a single incoherent "least effective dose" (1). A reasonable understanding of the RAIT paradigm would have led the authors to suggest robust prospective investigations aimed at refining our knowledge about the optimal prescribed activity for each of the 3 RAIT goals.

The paper by Molenaar et al. and prior similar reports fueled growing pressure on NMSs to lower prescribed activity ranges $(1,6-9)$. This trend has had a greater impact in countries where NMSs are not usually engaged in managing patients before and after RAIT. Such is generally the case in the United States, Canada, Finland, England, and Italy, to mention a few. In countries where NMSs are more involved in clinical decision making and patient follow-up, such as Germany and Switzerland, this trend has not been as impactful. The main determinant of the above dichotomy is differences in the respective national health systems and the assertiveness of NMSs in the clinical care of these patients. For example, the training of NMSs in the United States does not include experience with clinical follow-up and long-term management, unlike the training in Germany and Switzerland. Hence, the third lesson has to do with increasing our engagement in clinics, which should result in greater autonomy, greater responsibility, and ultimately a more fulfilling role for NMSs in RAIT decision making, increasing our value to patients and health-care systems.

The risks of radiation have certainly stoked considerable fear among patients. There is a scarcity of studies on patient perceptions about radiation treatment. One study from 1977 demonstrated that patients are often anxious about radiation treatment and have negative preconceptions (10). The medical profession has not significantly relieved this anxiety. A recent study showed that quality-of-life scores are lower for WDTC patients than for breast cancer patients (11), even though health-care professionals have always emphasized to the patients that WDTC has a high rate of cure and a 5-y overall survival of $98 \%$. Obviously, that approach has not been effective enough and there is space for improvement—space that can be successfully filled by clinically engaged NMSs.

Administering RAIT by using an empirically prescribed activity based on surgical pathology is akin to navigating a ship by looking at the tips of surrounding icebergs. The pre-RAIT thyroglobulin level provides additional information on the extent of WDTC, but routine application of pre-RAIT SPECT/CT allows for an even better understanding by providing such information as the amount of remnant normal tissue and the extent of regional or distant metastasis. In many NMS practices, pre-RAIT thyroglobulin levels and scintigraphy are not considered in decisions on which of the 3 RAIT goals to pursue and on what amount of activity to prescribe. In selecting the prescribed activity, it is critical to standardize pre-RAIT assessment, including the whole-body radioiodine scan, routine neck SPECT/CT, and thyroglobulin measurement, in order to advance our practice of RAIT into the conceptual framework of radiotheranostics (12). It is time for NMSs to decide whether to stay on the traditional course of being a technical assistant in RAIT or to change our training and practice in the way that enables a greater clinical decision making. Let us not forget that the Titanic sank not because the navigators did not see the iceberg but because the ship could not change its course.

\section{DISCLOSURE}

No potential conflict of interest relevant to this article was reported.

\section{EDITOR'S NOTE}

On January 17, 2018, Dr. Mark Tulchinsky sent a petition to Dr. Stephen A. Cannistra, Editor-in-Chief of JCO, asking that the article by Molenaar et al. (1) be retracted. This petition was supported and undersigned by 67 experts in nuclear medicine in 16 countries worldwide and included a rebuttal of the conclusions reached by the authors of that article. A reply by the authors was received. 
Individuals interested in these documents should contact Mark Tulchinsky directly at mark.tulchinsky@gmail.com.

\section{REFERENCES}

1. Molenaar RJ, Sidana S, Radivoyevitch T, et al. Risk of hematologic malignancies after radioiodine treatment of well-differentiated thyroid cancer. J Clin Oncol. December 18, 2017 [Epub ahead of print].

2. Haugen BR, Alexander EK, Bible KC, et al. 2015 American Thyroid Association management guidelines for adult patients with thyroid nodules and differentiated thyroid cancer: the American Thyroid Association Guidelines Task Force on Thyroid Nodules and Differentiated Thyroid Cancer. Thyroid. 2016;26:1-133.

3. Ruel E, Thomas S, Dinan M, Perkins JM, Roman SA, Sosa JA. Adjuvant radioactive iodine therapy is associated with improved survival for patients with intermediate-risk papillary thyroid cancer. J Clin Endocrinol Metab. 2015;100: 1529-1536.

4. Franklyn JA, Maisonneuve P, Sheppard M, Betteridge J, Boyle P. Cancer incidence and mortality after radioiodine treatment for hyperthyroidism: a population-based cohort study. Lancet. 1999;353:2111-2115.
5. Holm LE, Hall P, Wiklund K, et al. Cancer risk after iodine-131 therapy for hyperthyroidism. J Natl Cancer Inst. 1991;83:1072-1077.

6. Iyer NG, Morris LG, Tuttle RM, Shaha AR, Ganly I. Rising incidence of second cancers in patients with low-risk (T1N0) thyroid cancer who receive radioactive iodine therapy. Cancer. 2011;117:4439-4446.

7. Metso S, Auvinen A, Huhtala H, Salmi J, Oksala H, Jaatinen P. Increased cancer incidence after radioiodine treatment for hyperthyroidism. Cancer. 2007;109: 1972-1979.

8. Molenaar RJ, Pleyer C, Radivoyevitch T, et al. Risk of developing chronic myeloid neoplasms in well-differentiated thyroid cancer patients treated with radioactive iodine. Leukemia. 2018;32:952-959.

9. Rubino C, de Vathaire F, Dottorini ME, et al. Second primary malignancies in thyroid cancer patients. Br J Cancer. 2003;89:1638-1644.

10. Peck A, Boland J. Emotional reactions to radiation treatment. Cancer. 1977;40: $180-184$.

11. Applewhite MK, James BC, Kaplan SP, et al. Quality of life in thyroid cancer is similar to that of other cancers with worse survival. World J Surg. 2016;40: 551-561.

12. Jadvar H, Chen X, Cai W, Mahmood U. Radiotheranostics in cancer diagnosis and management. Radiology. 2018;286:388-400. 